\title{
Improving acetyl-CoA biosynthesis in Saccharomyces cerevisiae via the overexpression of pantothenate kinase and $\mathrm{PDH}$ bypass
}

Wenshan Liu, Bo Zhang and Rongrong Jiang*

\begin{abstract}
Background: Acetyl-CoA is an important precursor in Saccharomyces cerevisiae. Various approaches have been adopted to improve its cytosolic level previously with the emphasis on engineering the "acetyl-" part of acetyl-CoA. To the best of our knowledge, there have been no reports on engineering the "-CoA" part so far.

Results: In this study, we had tried to engineer S. cerevisiae from both the "-CoA" part via pantothenate kinase overexpression (PanK from S. cerevisiae, the rate-limiting enzyme for CoA synthesis) and the "acetyl-"part through PDH bypass introduction (ALD6 from S. cerevisiae and SeAcs ${ }^{L 641 P}$ from Salmonella enteric). A naringenin-producing reporter strain had been constructed to reflect cytosolic acetyl-CoA level as acetyl-CoA is the precursor of naringenin. It was found that PanK overexpression or PDH bypass introduction alone only led to a twofold or 6.74-fold increase in naringenin titer, but the combination of both (strain CENFPAA01) had resulted in 24.4-fold increase as compared to the control (strain CENF09) in the presence of $0.5 \mathrm{mM}$ substrate $p$-coumaric acid. The supplement of PanK substrate pantothenate resulted in another 19\% increase in naringenin production.
\end{abstract}

Conclusions: To greatly enhance acetyl-CoA level in yeast cytosol, it is feasible to engineer both the "acetyl-" part and the "-CoA" part simultaneously. Insufficient CoA supply might aggravate acetyl-CoA shortage and cause low yield of target product.

Keywords: Acetyl-CoA, Pantothenate kinase, Pyruvate dehydrogenase bypass, Naringenin production, Pantothenate, Saccharomyces cerevisiae

\section{Background}

The continuous use of fossil fuels has led to environment change. In recent years, people are seeking alternative energy resource to replace traditional fossil fuels [1]. Microbial conversion of renewable feedstock into fuels and chemicals has been intensively investigated [2]. Escherichia coli and Saccharomyces cerevisiae (S. cerevisiae), the most popular microbial factories, have been engineered for the production of valuable products [3, 4]. Compared with E. coli, yeast has unique advantages,

*Correspondence: rongrongjiang28@msn.com

School of Chemical \& Biomedical Engineering, Nanyang Technological University, 62 Nanyang Drive, Singapore 637459, Singapore such as post-translational modifications, capacity of expressing complex enzymes like P450s, less possibility of potential phage contamination [5-7]. Thus, it has been engineered to utilize various feedstocks to produce natural products and biofuels [8, 9].

Acetyl-CoA is the precursor of a wide range of bioproducts, including isoprenoids, polyketides, flavonoids, stilbenes, fatty acids and lipids, polyhydroxyalkanoates, and 1-butanol [1, 6, 10]. These products are mostly synthesized by consuming cytosolic acetyl-CoA. However, as acetyl-CoA in yeast is mainly generated in mitochondria from pyruvate through pyruvate dehydrogenase $(\mathrm{PDH})$ complex, it needs carnitine/acetyl-carnitine shuttle to be transported out of 
mitochondria [6, 11]. In order to enhance acetyl-CoA level in cytosol, PDH bypass has been introduced into S. cerevisiae (Fig. 1). PDH bypass is composed of pyruvate decarboxylase (PDC), acetaldehyde dehydrogenase (ACDH, such as Ald6), and acetyl-CoA synthetase (ACS) [12]: the acetaldehyde formed from pyruvates by PDC can be converted into acetate by $\mathrm{ACDH}$, or to ethanol by alcohol dehydrogenase (ADH), and acetylCoA can be generated from acetate by ACS. Specifically, endogenous ALD6, and endogenous ACS1/ACS2 or SeAcs ${ }^{L 641 P}$ from Salmonella enteric have been used in PDH bypass buildup [6, 13]. In addition, other strategies, such as overexpression of $A D H 2$ (ADH2 converts ethanol into acetaldehyde) $[6,13]$, knockout of $M L S 1$ and CIT2 (encoding malate synthase and citrate synthase, respectively) [6], and knockout of $A D H 1$ to limit ethanol production from acetaldehyde [14], have been reported to enhance cytosolic acetyl-CoA supply in yeast. Combination of these approaches has been used to improve target product yield such as $\alpha$-santalene [6]. But the combination may not always lead to significant increase in acetyl-CoA supply in yeast, for example, $\mathrm{PDH}$ bypass was introduced together with the overexpression of exogenous ATP-dependent citrate lyase (ACL) or PDH complex (cytosol), but little improvement was observed [7].
Previous studies on improving acetyl-CoA level in $S$. cerevisiae have been focused on engineering the "acetyl-" part of acetyl-CoA, but to the best of our knowledge, there have been no reports on engineering the "-CoA" part so far. Pantothenate kinase (PanK) is considered to be the rate-limiting enzyme for CoA synthesis, which catalyzes the phosphorylation of pantothenate [15] (Fig. 1). It was reported previously that the overexpression of $m P a n K 1 \beta$ (an isoform of PanK) in mammalian cells would trigger 13-fold increase in intracellular CoA content [16]. PanK overexpression in E. coli could also lead to tenfold increase in its intracellular CoA level and fivefold increase in its acetyl-CoA level [17]. Therefore, in this work, we tried to overexpress PanK encoding endogenous gene $C A B 1$ to increase acetyl-CoA level in $S$. cerevisiae, together with PDH bypass introduction (ALD6 from S. cerevisiae and SeAcs ${ }^{L 641 P}$ from $S$. enteric). In order to demonstrate cytosolic acetyl-CoA improvement in yeast, we had chosen naringenin as our model product, which takes acetyl-CoA as its precursor (Fig. 1). A naringenin pathway of three genes, i.e., 4-coumarate:CoA ligase (4CL), chalcone synthase (CHS), and flavanone isomerase (CHI), was integrated into yeast genome first. The introduction of PDH bypass alone led to 6.74-fold increase in naringenin titer in the presence of $0.5 \mathrm{mM}$ substrate. PanK overexpression further enhanced

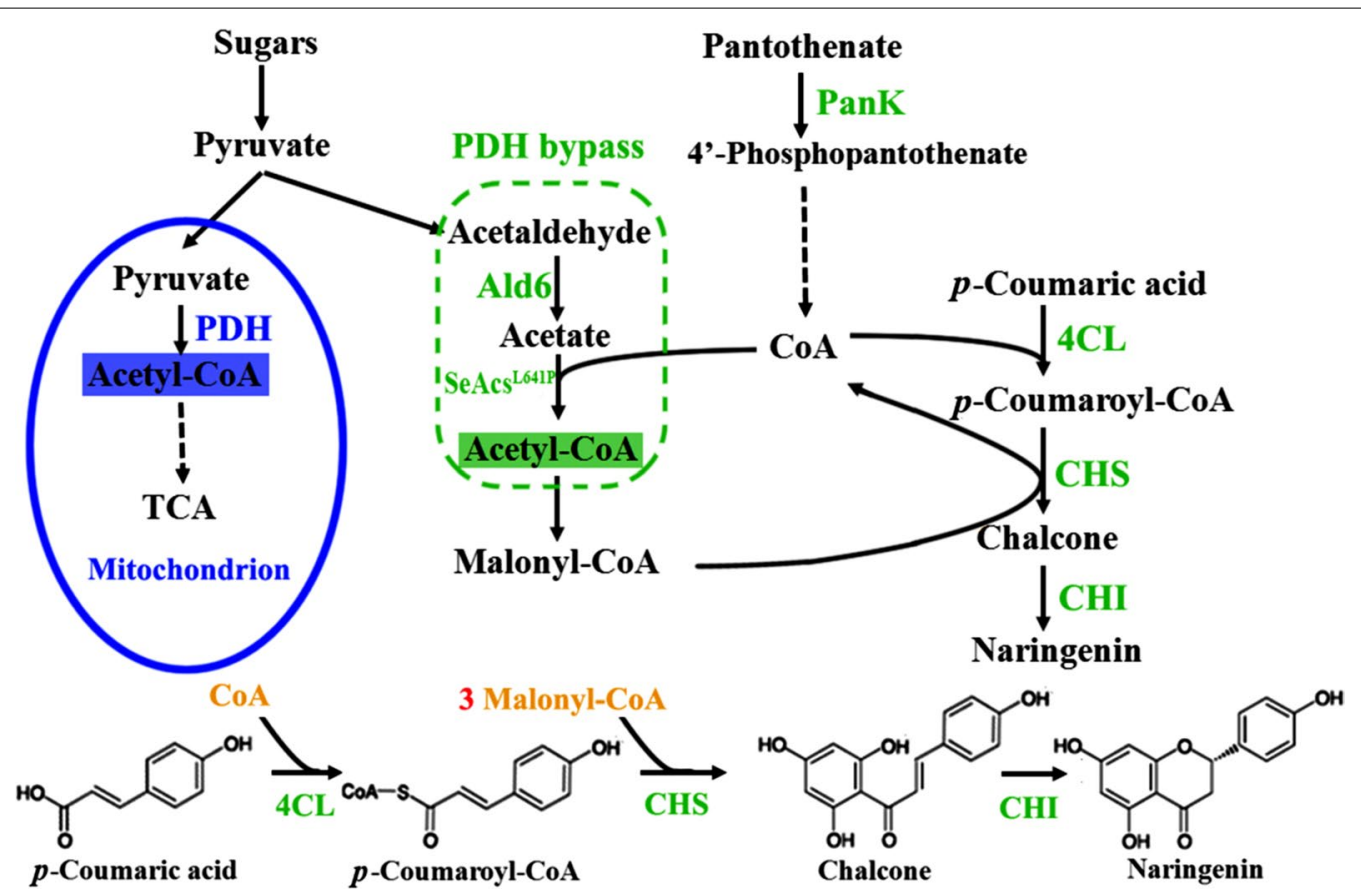

Fig. 1 Simplified overview of acetyl-CoA metabolism and associated naringenin synthesis pathway in S. cerevisiae. Acetyl-CoA is generated in different compartments in yeast, including mitochondria (blue), cytosol [25], and peroxisome (not shown). Three genes (4CL, CHS, and CHI) for naringenin synthesis were introduced into yeast genome. In addition, the rate-limiting enzyme of CoA synthesis, pantothenate kinase (PanK), and PDH bypass (ALD6 and SeACS ${ }^{L 41 P}$ ) were introduced into S. cerevisiae to improve its acetyl-CoA level in cytosol 
naringenin production by 24.4 -fold as compared to the control. The supplement of PanK substrate pantothenate resulted in another 19\% increase in naringenin production. An independent acetyl-CoA assay also confirmed the enhancement in cytosolic acetyl-CoA level in the engineered yeast strains.

\section{Methods}

\section{Plasmid and strain construction}

PanK encoding gene $C A B 1$ was amplified from S. cerevisiae BY4742 genome using primer pair 1\&2 (Table 1) with KAPA HIFI polymerase (KAPA Biosystems, Wilmington, MA, USA) and the PCR products were digested by SpeI and HindIII (all restriction enzymes in this study were from New England Biolab, Massachusetts, US) and inserted into the modified plasmid pRS426GAL1, which was reconstructed by removing its original $\mathrm{XhoI}$ and SalI sites (a pair of isocaudamer). The truncated HXT7 promoter amplified from yeast genome using primer pair 3 \& 4 was digested by SacI and SpeI and inserted between SacI and SpeI sites to replace the original GAL1 promoter. The new plasmid was named p426PanK.

TEF1p-ALD6-ADH1t expression cassette (TEF1p promoter, $A L D 6$ and $A D H 1 t$ terminator were all amplified from S. cerevisiae BY4742 genome) was constructed by overlap PCR using primers 5, 6, 7, 8, 9, and 10. Restriction endonuclease SacI, BamHI, XhoI, NheI, and NotI sites were introduced into the $5^{\prime}$-end of TEF1p using primer 5, while a SacI site was introduced into the $3^{\prime}$-end of ADH1t using primer 10. The cassette was digested with $S a c I$ and inserted into p426PanK to obtain plasmid p426PanK-Ald6. TDH3p-SeAcs ${ }^{\text {L641P }}$-PGIt expression cassette, with TDH3p promoter and PGIt terminator from S. cerevisiae and SeAcs ${ }^{L 641 P}$ from Salmonella enteric, was codon optimized and synthesized by GenScript (Piscataway, New Jersey, USA), which was digested by NheI and XhoI, and inserted into plasmid p426PanK-Ald6. The resulting plasmid was named p426PAA. The TEF1pALD6-ADH1t and TDH3p-SeAcs ${ }^{L 641 P}$-PGIt expression cassettes were cut from plasmid p426PAA by SacI and XhoI and inserted into plasmid pRS426Gal1 to generate plasmid p426AA. All constructed plasmids (Table 2) had been sent to DNA sequencing for confirmation.

To construct a naringenin-producing reporter strain, three genes involved in naringenin synthesis pathway were introduced into $S$. cerevisiae genome, namely 4-coumarate:CoA ligase (4CL) cDNA (GenBank: X13324) from Petroselinum crispum, chalcone synthase (CHS) cDNA (GenBank: AF233638) from Petunia hybrida, and flavanone isomerase $(\mathrm{CHI})$ cDNA (GenBank: Y00852) from Petunia hybrida. 4CL, CHI, and TEF1p-CHS-CYC1t expression cassettes were codon
Table 1 Primers in this study

\begin{tabular}{|c|c|}
\hline $\begin{array}{l}\text { Primer } \\
\text { no. }\end{array}$ & Sequence $\left(5^{\prime}-3^{\prime}\right)$ \\
\hline 1 & GTCAACTAGTATGCCGCGAATTACTCAAGAG \\
\hline 2 & GTACAAGCTTCTACGTACTTGTTTTTCTTAGTAG \\
\hline 3 & GTCAGAGCTCACTTCTCGTAGGAACAATTTC \\
\hline 4 & GTCAACTAGTTTTTTGATTAAAATTAAAAAAAC \\
\hline 5 & $\begin{array}{l}\text { ATCTGAGCTCGGATCCACTCGAGAGCTAGCAGCGGCCGC- } \\
\text { CACACACCATAGCTTCAA }\end{array}$ \\
\hline 6 & GTGTAGCTTAGTCATTTTGTAATTAAAACTTAG \\
\hline 7 & AGTTTTAATTACAAAATGACTAAGCTACACTTTGAC \\
\hline 8 & TCATAAGAAATTCGCTTACAACTTAATTCTGACAGC \\
\hline 9 & AGAATTAAGTTGTAAGCGAATTTCTTATGATTTATG \\
\hline 10 & CTATGAGCTCGATCCGTGTGGAAGAACG \\
\hline 11 & TTGTAATCGTTCTTCCACACGGATCTGGGGCCGTATACTTACATAT \\
\hline 12 & TGGAGCAACACAATCACCCATGTTTAGTTAATTATAGTTCGT \\
\hline 13 & ACGAACTATAATTAACTAAACATGGGTGATTGTGTTGCTCCA \\
\hline 14 & GTAAAGACATAAGAGATCCGCTTACTTTGGCAAATCACCAGA \\
\hline 15 & TCTGGTGATTTGCCAAAGTAAGCGGATCTCTTATGTCTTTAC \\
\hline 16 & $\begin{array}{l}\text { AAACATTTTGAAGCTATGGTGTGTGGGCATGCGAAGGAAAAT- } \\
\text { GAGA }\end{array}$ \\
\hline 17 & $\begin{array}{l}\text { GATGATAGTTGATTTCTATTCCAACAGTGAGTAAGGAAAGAGT- } \\
\text { GAGGAAC }\end{array}$ \\
\hline 18 & AACAGAAACTGGTGGAGACATTGTTTTATATTTGTTGTAAAAAG \\
\hline 19 & СTTTTTAACAACAAATATAAAACAATGTCTCCACCAGTTTCTGTT \\
\hline 20 & CATAAATCATAAGAAATTCGCTTAAACACCAATAACTGGAAT \\
\hline 21 & ATTCCAGTTATTGGTGTTTAAGCGAATTTCTTATGATTTATG \\
\hline 22 & TACTATATGTAAGTATACGGCCCCAGGATCCGTGTGGAAGAACGAT \\
\hline 23 & TCTCATTTTCCTTCGCATGCCCACACACCATAGCTTCAAAATG \\
\hline 24 & AAGGGTTGTCGACCTGCAGCGTAGCAAATTAAAGCCTTCGAGC \\
\hline 25 & TGGGACGCTCGAAGGCTTTAATTTGCTACGCTGCAGGTCGACAAC \\
\hline 26 & $\begin{array}{l}\text { CAACAACACCTGCTTCATCAGCTGTTACGACTCACTATAGGGA- } \\
\text { GACCG }\end{array}$ \\
\hline 27 & CTCGAGGGATATAGGAATCCTC \\
\hline 28 & $\begin{array}{l}\text { GTTCCTCACTCTTTCCTTACTCACTGTTGGAATAGAAATCAACTAT- } \\
\text { CATC }\end{array}$ \\
\hline 29 & CCGGTCTCCCTATAGTGAGTCGTAACAGCTGATGAAGCAGGTGT \\
\hline 30 & GAGAACTTCTAGTATATTCTGTATACCTAATATT \\
\hline
\end{tabular}

Table 2 Plasmids in this study

\begin{tabular}{|c|c|c|}
\hline Name & Description & Source \\
\hline pRS426GAL1 & $(2 \mu \cup R A 3)$ & [13] \\
\hline pUG6 & $\begin{array}{l}\text { Contains loxP-KanMX-loxP cassette for } \\
\text { knockout in yeast }\end{array}$ & {$[37]$} \\
\hline p426PanK & $P_{H X T 7}-C A B 1(2 \mu \cup R A 3)$ & This study \\
\hline p426PanK-Ald6 & $P_{H X T 7}-C A B 1 P_{\text {TEF } 1}-A L D 6(2 \mu U R A 3)$ & This study \\
\hline p426AA & $P_{\text {TEF }}-A L D 6 P_{\text {TDH3 }}-S e A c S^{L 641 P}(2 \mu$ URA3) & This study \\
\hline p426PAA & $\begin{array}{l}P_{H X T T}-C A B 1 P_{T E F 1}-A L D 6 P_{T D H 3}-\operatorname{SeACS}{ }^{L 641 P}(2 \mu \\
\quad \text { URA3) }\end{array}$ & This study \\
\hline
\end{tabular}


optimized and synthesized by GenScript (an additional file shows this in more detail, see Additional file 1). TEF2p-4CL-ADH2t and PGK1p-CHI-ADH1t expression cassettes were constructed by overlap PCR using primers 11-16 and 17-22. TEF1p-CHS-CYC1t expression cassette was amplified by primer $23 \& 24$. To facilitate the integration, three additional DNA fragments were also obtained: KanMX (selective marker) was amplified from plasmid pUG6 using primer $25 \& 26, \delta 1$ (248 bp in the $5^{\prime}$-end of $\delta$ sequence) and $\delta 2$ fragments (239 bp in the $3^{\prime}$-end of $\delta$ sequence) were amplified from yeast genome using primer $27 \& 28$ and primer $29 \& 30$, respectively. These six DNA fragments were assembled into the $\delta$ sites of $S$. cerevisiae CEN.PK2-1C chrome (MATa; ura352; trp1-289; leu2-3,112; his3 $\triangle 1$; MAL2-8C; SUC2) by a DNA assembler method [18]. The successfully assembled strain (CENF09) was confirmed by PCR with aforementioned primers (Table 3). Plasmid p426PanK was transformed into strain CEN.PK2-1C and CENF09 to create CENP01 and CENFP01, respectively, using the LiAc/SS carrier DNA/PEG method [19]. The same approach was used to transform plasmid p426AA into strain CEN.PK2$1 \mathrm{C}$ and CENF09 to generate CENAA01 and CENFAA01, respectively. Plasmid p426PAA was also transformed into strain CEN.PK2-1C and CENF09 to obtain CENPAA01 and CENFPAA01, respectively. In order to identify positive clones, plasmids were extracted with Zymoprep Yeast Plasmid Miniprep II Kit (Zymo Research, Irvine, $C A)$ and transformed into $E$. coli for verification by both restriction enzyme digestion and PCR.

\section{Media and growth conditions}

Escherichia coli DH5 $\alpha$ was used for cloning and cultured in Luria-Bertani [20] broth with $100 \mu \mathrm{g} / \mathrm{mL}$ ampicillin at $37^{\circ} \mathrm{C}$. Yeast cells were cultured in YPD media $(20 \mathrm{~g} / \mathrm{L}$ peptone, $10 \mathrm{~g} / \mathrm{L}$ yeast extract, and $20 \mathrm{~g} / \mathrm{L}$ glucose) at

Table 3 Strains in this study

\begin{tabular}{|c|c|c|}
\hline Name & Description & Source \\
\hline CEN.PK2-1C & $\begin{array}{l}\text { MATa; ura3-52; trp 1-289; leu2-3, 112; his3 } \triangle 1 \text {; } \\
\text { MAL2-8C; SUC2 }\end{array}$ & EUROSCARF \\
\hline CENF09 & $\begin{array}{l}\text { CEN.PK2-1C with naringenin synthesis } \\
\text { pathway }\left(P_{\text {TEFF- }}-4 C L P_{\text {TEF }}-C H S P_{P G K 1}-C H I\right) \\
\text { integrated into } \delta \text { sites in chromosome, } \\
\text { using Kan } M X \text { for selection }\end{array}$ & This study \\
\hline CENP01 & CEN.PK2-1C + p426PanK & This study \\
\hline CENFP01 & CENF09 + p426PanK & This study \\
\hline CENAA01 & CEN.PK2-1C + p426AA & This study \\
\hline CENFAA01 & CENF09 + p426AA & This study \\
\hline CENPAA01 & CEN.PK2-1C + p426PAA & This study \\
\hline CENFPAA01 & CENF09 + p426PAA & This study \\
\hline
\end{tabular}

$30{ }^{\circ} \mathrm{C}$. Recombinant yeast strains were screened and grown in YPD containing $200 \mu \mathrm{g} / \mathrm{mL}$ G418, or auxotrophic Complete Minimal medium (CM, $6.7 \mathrm{~g} / \mathrm{L}$ yeast nitrogen base without amino acids, $20 \mathrm{~g} / \mathrm{L}$ glucose, $150 \mathrm{mg} / \mathrm{L}$ valine, $20 \mathrm{mg} / \mathrm{L}$ adenine hemisulfate, $20 \mathrm{mg} / \mathrm{L}$ arginine- $\mathrm{HCl}, 30 \mathrm{mg} / \mathrm{L}$ lycine- $\mathrm{HCl}, 20 \mathrm{mg} / \mathrm{L}$ methionine, $200 \mathrm{mg} / \mathrm{L}$ threonine, $30 \mathrm{mg} / \mathrm{L}$ tyrosine, $50 \mathrm{mg} / \mathrm{L}$ phenylalanine, optionally supplemented with $100 \mathrm{mg} / \mathrm{L}$ leucine, $20 \mathrm{mg} / \mathrm{L}$ histidine, $20 \mathrm{mg} / \mathrm{L}$ uracile, and $20 \mathrm{mg} / \mathrm{L}$ tryptophane) at $30^{\circ} \mathrm{C}$.

\section{Naringenin fermentation and HPLC analysis}

Yeast colonies of CENF09, CENFP01, CENFAA01, and CENFPAA01 were pre-cultured in 5-mL CM medium in 50 -mL tubes overnight at $30{ }^{\circ} \mathrm{C}, 225 \mathrm{rpm}$, respectively. The pre-culture was then diluted into fresh $20-\mathrm{mL} \mathrm{CM}$ medium in $250-\mathrm{mL}$ flasks to a final $\mathrm{OD}_{600}$ of 0.05 , respectively. Fermentation was carried out at $30^{\circ} \mathrm{C}, 225 \mathrm{rpm}$ for $96 \mathrm{~h}$, with substrate $p$-coumaric acid (Sigma-Aldrich, St. Louis, MO, USA) concentration at $0.5 \mathrm{mM}$. In addition, the best acetyl-CoA-producing strain was also tested at a series of $p$-coumaric acid concentration: $0.05,0.1,0.2$, $0.3,0.4$, and $0.5 \mathrm{mM}$.

The fermentation broth was centrifuged at 12,000 rpm for $10 \mathrm{~min}$. Samples from each supernatant were taken for HPLC analysis on a XDB-C18 column (Agilent, Santa Clara, USA). Compounds were separated by elution with acetonitrile-water gradient at $1.0 \mathrm{ml} / \mathrm{min}$ as described previously [21]. Naringenin standard (ACROS organics, New Jersey, USA) and naringenin from the samples were detected by its UV absorbance at $290 \mathrm{~nm}$.

\section{Acetyl-CoA measurement}

Acetyl-CoA was analyzed according to a previously described method [7]. Yeast colonies of CENP01, CENAA01, and CENPAA01 and wild-type CEN.PK2$1 \mathrm{C}$ were pre-cultured in $5-\mathrm{mL} \mathrm{CM}$ medium in $50-\mathrm{mL}$ tubes overnight at $30{ }^{\circ} \mathrm{C}, 225 \mathrm{rpm}$. The pre-cultures were diluted into fresh 50-mL CM medium to a final $\mathrm{OD}_{600}$ of 0.05 . Cells were harvested during mid-log phase by centrifugation at 12,000 rpm for $5 \mathrm{~min}$. 10-mL pre-chilled $\left(-80{ }^{\circ} \mathrm{C}\right)$ methanol was added to quench cell metabolism and centrifuged at 12,000 rpm for $5 \mathrm{~min}$ to remove the supernatant. $2 \mathrm{~mL}$ boiling ethanol was added to cell pellets and the mixture was treated thoroughly by glass beads for $5 \mathrm{~min}$ (vortex) to release intracellular metabolites. The supernatant was vacuum dried after

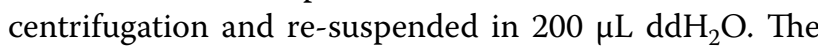
resulting solution containing acetyl-CoA was analyzed by an Acetyl-CoA Assay Kit (Sigma-Aldrich, St. Louis, MO, USA). Acetyl-CoA concentration obtained was an average of biological duplicates, normalized by dry cell weight. 


\section{Pantothenate effect on naringenin production}

CENFPAA01 was pre-cultured in $5 \mathrm{~mL} \mathrm{CM}$ medium in 50 -mL tubes overnight at $30{ }^{\circ} \mathrm{C}, 225 \mathrm{rpm}$. The precultures were diluted into fresh $20 \mathrm{~mL} C M$ medium containing $0.5 \mathrm{mM} p$-coumaric acid substrate to a final $\mathrm{OD}_{600}$ of 0.05. $50 \mathrm{mM}$ pantothenate (Sigma-Aldrich, St. Louis, MO, USA) stock solution was added to the liquid medium to achieve a final pantothenate concentration of 10,20 , and $50 \mu \mathrm{M}$, respectively. Fermentation was carried out at $30{ }^{\circ} \mathrm{C}, 225 \mathrm{rpm}$ for $96 \mathrm{~h}$. Samples were taken from each fermentation broth for naringenin measurement.

\section{Results}

\section{Construction of naringenin-producing reporter strain}

The amount of acetyl-CoA, an important precursor, is under dynamic changes due to continuous generation and consumption processes, and hence it is difficult to measure accurately $[22,23]$. Since acetyl-CoA is an important precursor for naringenin, naringenin pathway was introduced into $S$. cerevisiae as a reporter of its cytosolic acetyl-CoA level. Naringenin can be synthesized from $p$-coumaric acid in plants, which is catalyzed by three enzymes, 4CL, CHS, and CHI (Fig. 1). $4 \mathrm{CL}$ catalyzes the ligation of $\mathrm{CoA}$ and $p$-coumaric acid to generate $p$-coumaroyl-CoA. CHS is responsible for the condensation of three molecules of malonyl-CoA, which are generated from 3 molecules acetyl-CoA, and $p$-coumaroyl-CoA to produce chalcone. Lastly, $\mathrm{CHI}$ catalyzes the isomerization of chalcone to generate naringenin. Three molecules of acetyl-CoA are consumed in total for one molecule of naringenin generated. In this work, in order to construct a stable reporter strain, $4 C L$ gene from P. crispum, CHS and CHI genes from P. hybrida were codon optimized and synthesized for DNA assembly. All three genes were integrated into yeast genome by a single transformation step to generate strain CENF09. Constitutive promoters TEF1p, TEF2 $p$, and PGK1p were chosen for $C H S, 4 C L$, and $C H I$ transcription, respectively. As a result, strain CENF09 could generate $0.43 \mathrm{mg} / \mathrm{L}$ naringenin with $0.5 \mathrm{mM} p$-coumaric acid present (Fig. 2).

\section{Enhancing CoA/acetyl-CoA supply with PanK overexpression}

CoA was synthesized from pantothente, cysteine and ATP. For the first step, pantothenate is phosphorylated to 4'-phosphopantetheine by an ATP-dependent pantothenate kinase (Pank). 4'-phosphopantetheine reacts with cysteine to form $4^{\prime}$-phosphopantothenoylcysteine, which is subsequently decarboxylated to generate $4^{\prime}$-phosphopantetheine, which is changed into dephospho-CoA that is finally phosphorylated into CoA. The reaction catalyzed by pantothenate kinase is the key and rate-limiting step. PanK is encoded by CAB1 in S. cerevisiae, which is

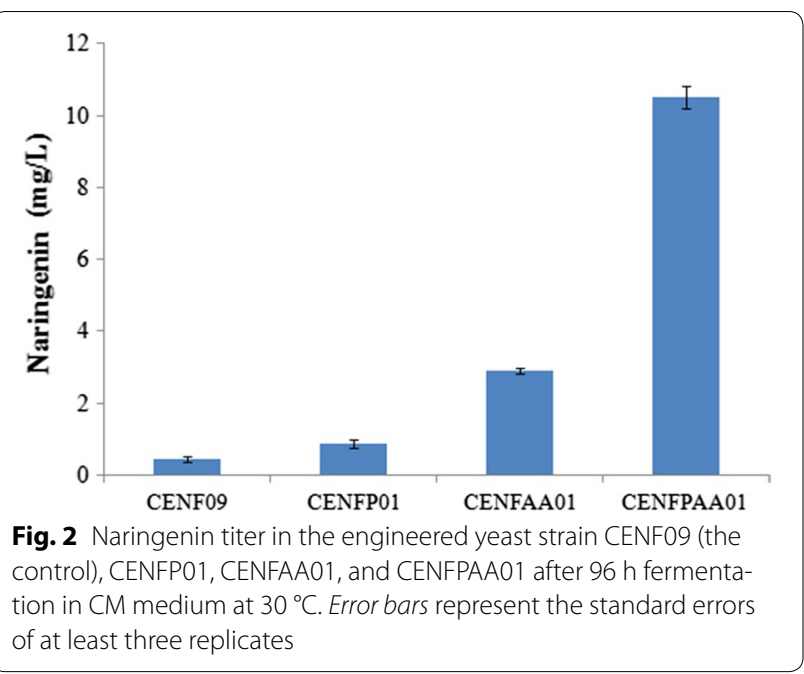

reported to be transcribed at low level [15]. To improve CoA synthesis, $C A B 1$ was overexpressed under a strong constitutive promoter (truncated HXT7 promoter) in CENF09 to create strain CENFP01, and the naringenin titer was found to be $0.88 \mathrm{mg} / \mathrm{L}$ in the presence of $0.5 \mathrm{mM}$ substrate, about two-fold that of the control CENF09 (Fig. 2).

\section{Introduction of PDH bypass to further improve acetyl-CoA level}

A PDH bypass, which generates acetyl-CoA from acetaldehydes via Ald6 from S. cerevisiae and mutant ACS from S. enteric (SeAcs ${ }^{\mathrm{L} 641 \mathrm{P}}$ ), was reported previously to enhance acetyl-CoA supply for amorphadiene [13] and $\alpha$-santalene [6]. Due to the difficulty to overexpress PDC complex, ALD6 gene (S. cerevisiae) and SeAcs ${ }^{L 641 P}$ (S. enteric) were overexpressed in this study for PDH bypass construction, under constitutive TEF1 and TDH3 promoter in plasmid p426AA, which was introduced into yeast to create strain CENFAA01. It had demonstrated better naringenin production $-2.90 \mathrm{mg} / \mathrm{L}$ naringenin with $0.5 \mathrm{mM} p$-coumaric acid present, 6.74-fold increase as compared to that of the control CENF09 (Fig. 2).

In order to further improve intercellular acetyl-CoA level in yeast, PDH bypass and PanK were both introduced into CENF09 to generate strain CENFPAA01. A significant enhancement in naringenin titer was observed in CENFPAA01, $10.51 \mathrm{mg} / \mathrm{L}$, which was 24.44 -fold increase as compared to that of the control CENF09, 11.94-fold of CENFP01, and 3.63-fold of CENFAA01 (Fig. 2).

We had also tracked cell growth of CENF09, CENFP01, CENFAA01, and CENFPAA0 during naringenin production. As shown in Fig. 3a, there is no significant differences on the growth after $120 \mathrm{~h}$ culture in CM medium among strain CENF09, CENFP01, and CENFAA01, 

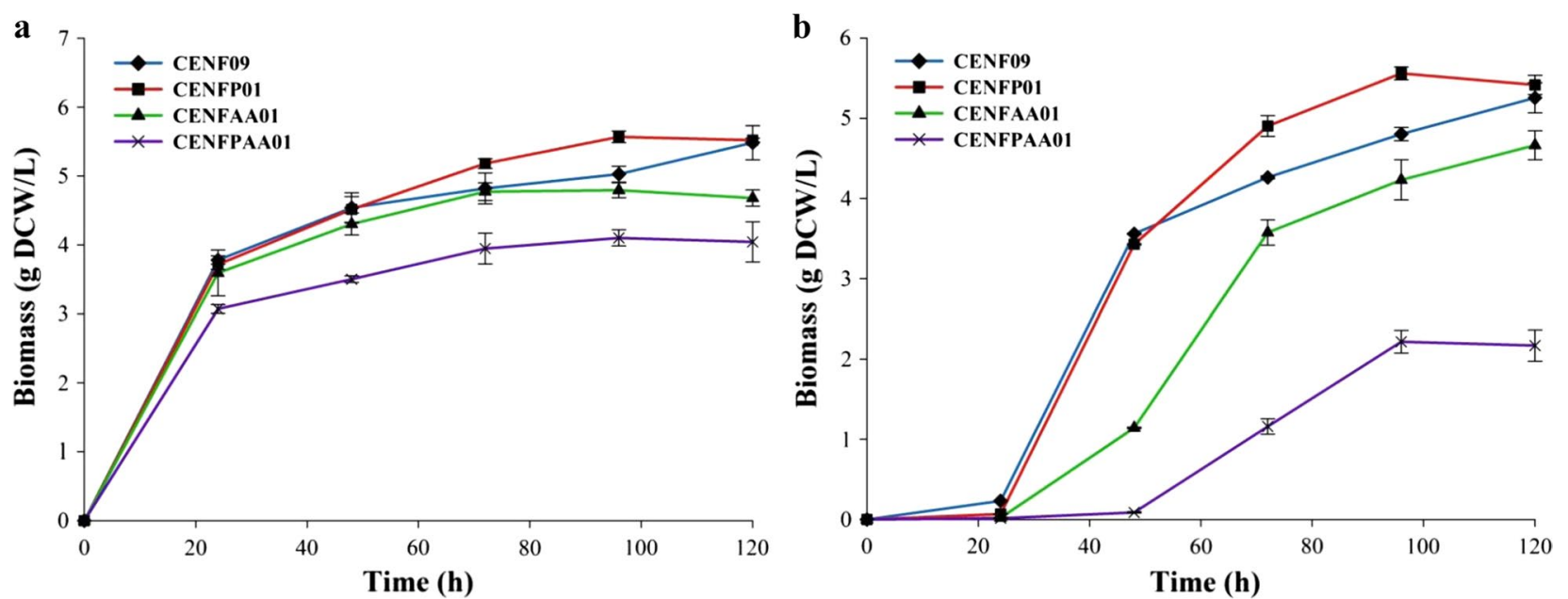

Fig. 3 Growth profile of naringenin-producing strains in CM medium (a) without p-coumaric acid; $\mathbf{b}$ with $0.5 \mathrm{mM}$ p-coumaric acid. Error bars represent standard errors of at least three replicates

whereas CENFPAA01 showed much less biomass accumulation, which means the introduction of PanK or PDH bypass alone did not affect cell growth very much, but the co-expression of PanK and PDH bypass had caused heavy metabolic burden to yeast cells. Substrate $p$-coumaric acid itself also demonstrated inhibitory effects on cell growth during the first $24 \mathrm{~h}$ as shown in Fig. 3b. $p$-coumaric acid almost did not change the final biomass of CENF09 and CENFP01 after $120 \mathrm{~h}$, while CENFAA01 grew slower than without $p$-coumaric acid. Especially, CENFPAA01 in CM with $0.5 \mathrm{mM} p$-coumaric acid grew much slower than that of without $p$-coumaric acid. Moreover, if taking cell growth into account, naringenin titer in the best acetyl-CoA-producing strain CENFPAA01 was 54-fold that of CENF09, 30-fold of CENFP01, and sevenfold of CENFAA01, in the presence of $0.5 \mathrm{mM}$ $p$-coumaric acid.

Since CENFPAA01 was the best naringenin- and acetyl-CoA-producing strain obtained, it was utilized for substrate optimization. As shown in Fig. 4, naringenin production increased with $p$-coumaric acid concentration and reached its optimum $(\sim 10.50 \mathrm{mg} / \mathrm{L})$ at $0.4-$ $0.5 \mathrm{mM} p$-coumaric acid. As other research groups had also reported their naringenin production with $0.5 \mathrm{mM}$ $p$-coumaric acid $[21,24,25]$, we set substrate concentration at $0.5 \mathrm{mM}$ in this work.

\section{Acetyl-CoA assay}

To further confirm that the rise in naringenin production was due to acetyl-CoA increase in yeast, we had determined acetyl-CoA concentration in native strain CEN. PK2-1C and three non-naringenin-producing strains (CENP01, CENAA01, and CENPAA01) with Acetyl-CoA Assay Kit, of which a CoA quencher would help remove free CoA background. As displayed by Fig. 5, the overexpression of PanK (CENP01) or PDH bypass (CENAA01) alone did not show significant acetyl-CoA level improvement as compared to native strain CEN.PK2-1C. However, when PanK and PDH bypass were co-expressed in CENPAA01, acetyl-CoA level was greatly enhanced by $~$ threefold as compared to CEN.PK2-1C, which was in agreement with aforementioned findings on naringenin generation (Fig. 2).

\section{Pantothenate effect on intracellular acetyl-CoA level}

Pantothenate, the substrate for PanK and precursor for $\mathrm{CoA}$, is directly related with acetyl-CoA biosynthesis in S. cerevisiae. Since PanK is the rate-limiting step for CoA synthesis and previous reports have shown that pantothenate supplement could help increase CoA/acetyl-CoA level in mammalian and E. coli cells $[16,17]$, pantothenate concentration in the fermentation broth was optimized in this work for naringenin production. As shown in Fig. 6, the titer rose with pantothenate addition when pantothenate concentration increased from 10 to $50 \mu \mathrm{M}$. Naringenin titer was at its maximum in the presence of $50 \mu \mathrm{M}$ pantothenate $(12.49 \mathrm{mg} / \mathrm{L})$, just a bit higher than that of $20 \mu \mathrm{M}(12.22 \mathrm{mg} / \mathrm{L}), \sim 19 \%$ increase as compared to the case without $(10.51 \mathrm{mg} / \mathrm{L})$, which also suggests that increasing CoA supply is crucial for improving cytosolic acetyl-CoA level in yeast.

\section{Discussion}

In this work, PDH bypass and PanK, the rate-limiting enzyme for CoA synthesis, were both introduced into a naringenin-producing reporter strain to demonstrate its cytosolic acetyl-CoA level improvement. The best engineered strain CENFPAA01 showed naringenin titer 


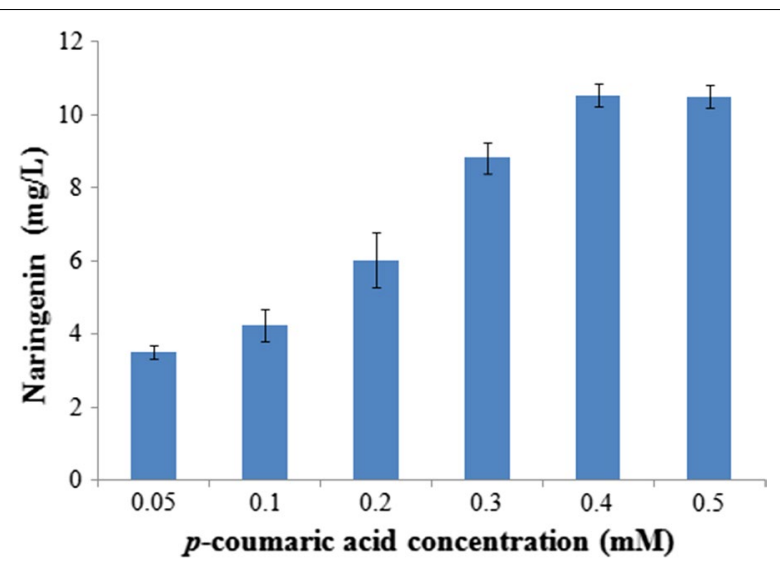

Fig. 4 Naringenin production in CENFPAA01 with various substrate p-coumaric acid concentration, $96 \mathrm{~h}$ fermentation in CM medium at $30^{\circ} \mathrm{C}$. Error bars represent the standard errors of at least three replicates
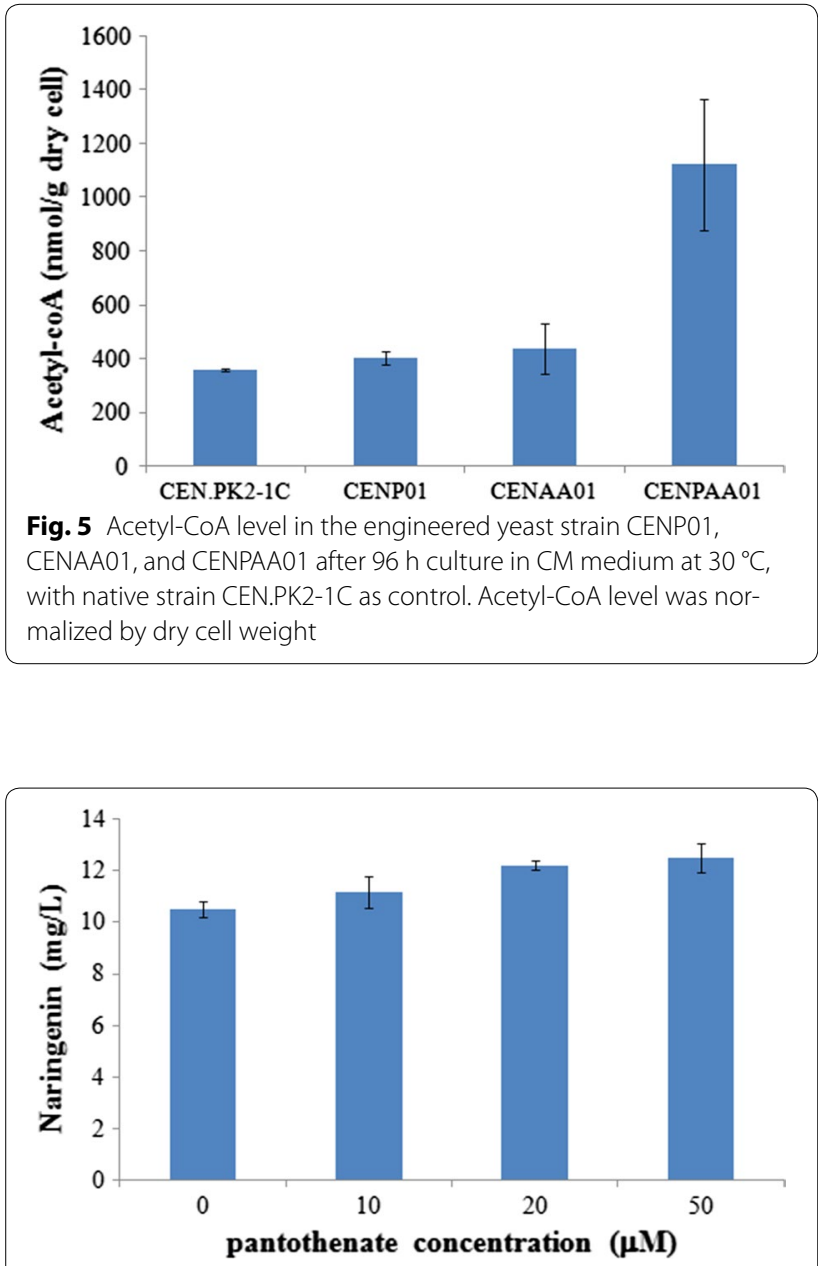

Fig. 6 Pantothenate effect on naringenin production in CENFPAA01, $96 \mathrm{~h}$ fermentation in $\mathrm{CM}$ medium at $30^{\circ} \mathrm{C}$. Error bars represent the standard errors of at least three repeats. PA pantothenate at $10.51 \mathrm{mg} / \mathrm{L}$ in the presence of $0.5 \mathrm{mM} p$-coumaric acid, which was 24.4-fold increase of that of the control CENF09. PanK substrate, pantothenate supplement has led to another $19 \%$ increase in naringenin production, and the final titer was further increased to $12.49 \mathrm{mg} / \mathrm{L}$, which suggests that enhancing CoA supply could help improve acetyl-CoA level in yeast.

We found that to greatly enhance acetyl-CoA level in yeast cytosol, both the "acetyl-" part and the "-CoA" part have to be engineered simultaneously. The introduction of PanK or PDH bypass alone only showed moderate enhancement in naringenin production, namely twofold and 6.74-fold, respectively. However, when both were introduced into strain CENFPAA01, naringenin titer was dramatically improved by 24.4 -fold as compared to the control CENF09. These findings were double confirmed by an independent acetyl-CoA assay (Fig. 5). Previous studies on ACL and PDH complex overexpression have successfully improved target products with acetyl-CoA as precursors [26, 27]. However, ACL catalyzes the formation of acetyl-CoA and oxaloacetate from citrate and CoA in the presence of ATP in cytoplasm. PDH complex overexpression mainly focuses on increasing the acetyl- part. Thus, the supply of CoA might affect the final acetyl-CoA production. The idea is in agreement with previous findings that the introduction of PDH bypass alone could not improve cytosolic acetyl-CoA level greatly in yeast. Chen et al. showed that PDH bypass could only enhance $\alpha$-santalene titer by $50 \%$ in S. cerevisiae $[6,11]$. Compared with above methods, our method is to ensure the balance between CoA and acetyl- part and maximize the acetylCoA production. The "-CoA" engineering approach via PanK overexpression discussed here probably can also be combined with other "acetyl-" engineering methods to further help increase cytosolic acetyl-CoA supply in yeast.

The maximum naringenin titer reported here is $12.49 \mathrm{mg} / \mathrm{L}$, better than the titer from a previous report of introducing phenylalanine ammonia lyase [28] for de novo synthesis of naringenin $(5.8 \mathrm{mg} / \mathrm{L})$ [29]. Our titer was still lower than the naringenin titer $(28.3 \mathrm{mg} / \mathrm{L})$ reported by Koffas group, which was achieved by adding substrate $p$-coumaric acid every $13 \mathrm{~h}$ to the culture in five equal doses [30]. To the best of our knowledge, all studies on flavonoid production in yeast used expression plasmids containing GAL1 or GAL10 promoter [29-31]. However, the repetitive homologous sequence of promoters may cause high possibility of gene deletion after rounds of subcultures [32, 33]. In this work, we had constructed a stable naringenin-producing strain for the evaluation of acetyl-CoA level in yeast- $4 C L, C H S$, and $\mathrm{CHI}$ for naringenin synthesis were regulated by constitutive promoters and integrated into yeast genome. 
Yan et al. [34] added substrate $p$-coumaric acid every $13 \mathrm{~h}$ to avoid its toxicity to yeast cells. Interestingly, we had also found that the control CENF09 had higher naringenin production at low $p$-coumaric acid concentration, namely $3.55 \mathrm{mg} / \mathrm{L}$ with $0.1 \mathrm{mM}$ substrate and $0.43 \mathrm{mg} / \mathrm{L}$ with $0.5 \mathrm{mM}$. One possible explanation for this phenomenon could be the insufficient acetyl-CoA supply when $p$-coumaric acid concentration increased from 0.1 to $0.5 \mathrm{mM}$. CHS was reported to be the rate-limiting enzyme for flavonoid synthesis in oat primary leaves [34], and the flavonoid production could be regulated by CHS expression in Juglans nigra and cucumber plants [35, 36]. As such, if acetyl-CoA supply is insufficient, CHS reaction would slow down and less naringenin would be generated. At the same time, larger amount of CoA is consumed to produce $p$-coumaroyl-CoA with $p$-coumaric acid concentration increases from 0.1 to $0.5 \mathrm{mM}$. Hence, insufficient CoA supply might aggravate acetyl-CoA shortage for the $\mathrm{CHS}$ step and led to lower naringenin titer.

\section{Conclusions}

In this study, we have demonstrated that the combination of PDH bypass and PanK overexpression would greatly enhance acetyl-CoA level in S. cerevisiae cytosol. It is the first report to engineer both the "acetyl-" part and the "-CoA" part simultaneously in yeast to improve acetylCoA production. Taking naringenin as sample product, the acetyl-CoA increase has led to 24.4-fold increase in its titer. We hope this approach could also help improve other chemical production in yeast, which takes acetylCoA as its precursor.

\section{Additional file}

Additional file 1. Additional materials

\begin{abstract}
Abbreviations
PanK: pantothenate kinase; PDH: pyruvate dehydrogenase; PDC: pyruvate decarboxylase; ALD6: acetaldehyde dehydrogenase; ACS: acetyl-CoA synthetase; $\mathrm{ADH}$ : alcohol dehydrogenase; 4CL: 4-coumarate:CoA ligase; CHS: chalcone synthase; $\mathrm{CHI}$ : flavanone isomerase.
\end{abstract}

\section{Authors' contributions}

LW and ZB conducted the experiments. LW, ZB, and JR made the design of this study and drafted the manuscript. $Z B$ and JR revised the manuscript. All authors were involved in the intellectual aspects of the study. All authors read and approved the final manuscript.

\section{Acknowledgements}

We would also like to thank Prof. Michel Ghislain from Université catholique de Louvain for providing us plasmid pRS426GAL1 and pUG6.

\section{Competing interests}

The authors declare that they have no competing interests.

\section{Funding}

This work was supported by Competitive Research Program (NRF-

CRP5-2009-03) from National Research Foundation, Singapore.
Received: 24 May 2016 Accepted: 9 February 2017

Published online: 17 February 2017

\section{References}

1. Swidah R, Wang H, Reid PJ, Ahmed HZ, Pisanelli AM, Persaud KC, et al. Butanol production in S. cerevisiae via a synthetic ABE pathway is enhanced by specific metabolic engineering and butanol resistance. Biotechnol Biofuels. 2015;8:97

2. Lian J, Zhao H. Recent advances in biosynthesis of fatty acids derived products in Saccharomyces cerevisiae via enhanced supply of precursor metabolites. J Ind Microbiol Biotechnol. 2015:42:437-51.

3. Du J, Shao ZY, Zhao HM. Engineering microbial factories for synthesis of value-added products. J Ind Microbiol Biotechnol. 2011;38:873-90.

4. Woolston BM, Edgar S, Stephanopoulos G. Metabolic engineering: past and future. Annu Rev Chem Biomol Eng. 2013;4:259-88.

5. Nielsen J, Jewett MC. Impact of systems biology on metabolic engineering of Saccharomyces cerevisiae. FEMS Yeast Res. 2008;8:122-31.

6. Chen Y, Daviet L, Schalk M, Siewers V, Nielsen J. Establishing a platform cell factory through engineering of yeast acetyl-CoA metabolism. Metab Eng. 2013:15:48-54.

7. Lian J, Si T, Nair NU, Zhao H. Design and construction of acetyl-CoA overproducing Saccharomyces cerevisiae strains. Metab Eng. 2014;24:139-49.

8. Liu LQ, Redden H, Alper HS. Frontiers of yeast metabolic engineering: diversifying beyond ethanol and Saccharomyces. Curr Opin Biotechnol. 2013;24:1023-30.

9. Hong KK, Nielsen J. Metabolic engineering of Saccharomyces cerevisiae: a key cell factory platform for future biorefineries. Cell Mol Life Sci. 2012;69:2671-90.

10. Binder S, Siedler S, Marienhagen J, Bott M, Eggeling L. Recombineering in Corynebacterium glutamicum combined with optical nanosensors: a general strategy for fast producer strain generation. Nucleic Acids Res. 2013;41:6360-9.

11. Strijbis K, Distel B. Intracellular acetyl unit transport in fungal carbon metabolism. Eukaryot Cell. 2010;9:1809-15.

12. Remize F, Andrieu E, Dequin S. Engineering of the pyruvate dehydrogenase bypass in Saccharomyces cerevisiae: role of the cytosolic $\mathrm{Mg}^{2+}$ and mitochondrial $\mathrm{K}^{+}$acetaldehyde dehydrogenases Ald6p and Ald4p in acetate formation during alcoholic fermentation. Appl Environ Microbiol. 2000;66:3151-9.

13. Shiba Y, Paradise EM, Kirby J, Ro DK, Keasling JD. Engineering of the pyruvate dehydrogenase bypass in Saccharomyces cerevisiae for high-level production of isoprenoids. Metab Eng. 2007:9:160-8.

14. Li X, Guo D, Cheng Y, Zhu F, Deng Z, Liu T. Overproduction of fatty acids in engineered Saccharomyces cerevisiae. Biotechnol Bioeng. 2014;111:1841-52.

15. Olzhausen J, Schubbe S, Schuller HJ. Genetic analysis of coenzyme A biosynthesis in the yeast Saccharomyces cerevisiae: identification of a conditional mutation in the pantothenate kinase gene CAB1. Curr Genet. 2009;55:163-73.

16. Rock CO, Calder RB, Karim MA, Jackowski S. Pantothenate kinase regulation of the intracellular concentration of coenzyme A. J Biol Chem. 2000;275:1377-83.

17. Vadali RV, Bennett GN, San KY. Cofactor engineering of intracellular CoA/ acetyl-CoA and its effect on metabolic flux redistribution in Escherichia coli. Metab Eng. 2004;6:133-9.

18. Shao ZY, Zhao H, Zhao HM. DNA assembler, an in vivo genetic method for rapid construction of biochemical pathways. Nucleic Acids Res. 2009;37:e16.

19. Gietz RD, Schiestl RH. Frozen competent yeast cells that can be transformed with high efficiency using the LiAc/SS carrier DNA/PEG method. Nat Protoc. 2007;2:1-4.

20. Qi LS, Larson MH, Gilbert LA, Doudna JA, Weissman JS, Arkin AP, Lim WA. Repurposing CRISPR as an RNA-guided platform for sequence-specific control of gene expression. Cell. 2013;152:1173-83.

21. Leonard $E$, Yan $Y$, Lim KH, Koffas MA. Investigation of two distinct flavone synthases for plant-specific flavone biosynthesis in Saccharomyces cerevisiae. Appl Environ Microbiol. 2005;71:8241-8. 
22. Park JW, Jung WS, Park SR, Park BC, Yoon YJ. Analysis of intracellular short organic acid-coenzyme A esters from actinomycetes using liquid chromatography-electrospray ionization-mass spectrometry. J Mass Spectrom. 2007;42:1136-47.

23. Steen EJ, Chan R, Prasad N, Myers S, Petzold CJ, Redding A, et al. Metabolic engineering of Saccharomyces cerevisiae for the production of n-butanol. Microb Cell Fact. 2008;7:36.

24. Chemler JA, Yan Y, Leonard E, Koffas MA. Combinatorial mutasynthesis of flavonoid analogues from acrylic acids in microorganisms. Org Lett. 2007;9:1855-8.

25. Naesby M, Nielsen SV, Nielsen CA, Green T, Tange TO, Simón E, et al. Yeast artificial chromosomes employed for random assembly of biosynthetic pathways and production of diverse compounds in Saccharomyces cerevisiae. Microb Cell Fact. 2009;8:45.

26. Tang $X$, Feng $H$, Chen WN. Metabolic engineering for enhanced fatty acids synthesis in Saccharomyces cerevisiae. Metab Eng. 2013;16:95-102.

27. Cardenas J, Da Silva NA. Engineering cofactor and transport mechanisms in Saccharomyces cerevisiae for enhanced acetyl-CoA and polyketide biosynthesis. Metab Eng. 2016;36:80-9.

28. Varma A, Palsson BO. Metabolic flux balancing-basic concepts, scientific and practical use. Nat Biotechnol. 1994;12:994-8.

29. Jiang HX, Wood KV, Morgan JA. Metabolic engineering of the phenylpropanoid pathway in Saccharomyces cerevisiae. Appl Environ Microbiol. 2005;71:2962-9.

30. Yan YJ, Kohli A, Koffas MA. Biosynthesis of natural flavanones in Saccharomyces cerevisiae. Appl Environ Microbiol. 2005;71:5610-3.
31. Trantas E, Panopoulos N, Ververidis F. Metabolic engineering of the complete pathway leading to heterologous biosynthesis of various flavonoids and stilbenoids in Saccharomyces cerevisiae. Metab Eng. 2009;11:355-66.

32. Hua SB, Qiu M, Chan E, Zhu L, Luo Y. Minimum length of sequence homology required for in vivo cloning by homologous recombination in yeast. Plasmid. 1997;38:91-6.

33. Raymond CK, Sims EH, Olson MV. Linker-mediated recombinational subcloning of large DNA fragments using yeast. Genome Res. 2002;12:190-7.

34. Knogge W, Schmelzer E, Weissenbock $G$. The role of chalcone synthase in the regulation of flavonoid biosynthesis in developing oat primary leaves. Arch Biochem Biophys. 1986;250:364-72.

35. Beritognolo I, Magel E, Abdel-Latif A, Charpentier JP, Jay-Allemand C, Breton $C$. Expression of genes encoding chalcone synthase, flavanone 3-hydroxylase and dihydroflavonol 4-reductase correlates with flavanol accumulation during heartwood formation in Juglans nigra. Tree Physiol. 2002;22:291-300

36. Fofana B, Benhamou N, McNally DJ, Labbé C, Séguin A, Bélanger RR. Suppression of induced resistance in cucumber through disruption of the flavonoid pathway. Phytopathology. 2005;95:114-23.

37. Güldener U, Heck S, Fielder T, Beinhauer J, Hegemann JH. A new efficient gene disruption cassette for repeated use in budding yeast. Nucleic Acids Res. 1996;24:2519-24.

\section{Submit your next manuscript to BioMed Central and we will help you at every step:}

- We accept pre-submission inquiries

- Our selector tool helps you to find the most relevant journal

- We provide round the clock customer support

- Convenient online submission

- Thorough peer review

- Inclusion in PubMed and all major indexing services

- Maximum visibility for your research

Submit your manuscript at www.biomedcentral.com/submit 